\title{
Classical Interaction of a Magnet and a Point Charge: The Shockley-James Paradox
}

\author{
Timothy H. Boyer \\ Department of Physics, City College of the City \\ University of New York, New York, New York 10031
}

\begin{abstract}
It is pointed out that Coleman and Van Vleck make a major blunder in their discussion of the Shockly-James paradox by designating relativistic "hidden mechanical momentum" as the basis for resolution of the paradox. This blunder has had a wide influence in the current physics literature, including erroneous work on the Shockley-James paradox, on Mansuripur's paradox, on the motion of a magnetic moment, on the Aharonov-Bohm phase shift, and on the Aharonov-Casher phase shift. Although hidden mechanical momentum is indeed dominant for non-interacting particles moving in a closed orbit under the influence of an external electric field, the attention directed toward hidden mechanical momentum represents a fundamental misunderstanding of the classical electromagnetic interaction between a multiparticle magnet and an external point charge. In the interacting multiparticle situation, the external charge induces an electrostatic polarization of the magnet which leads to an internal electromagnetic momentum in the magnet where both the electric and magnetic fields for the momentum are contributed by the magnet particles. This internal electromagnetic momentum for the interacting multiparticle situation is equal in magnitude and opposite in direction compared to the familiar external electromagnetic momentum where the electric field is contributed by the external charged particle and the magnetic field is that due to the magnet. In the present article, the momentum balance of the Shockley-James situation for a system of a magnet and a point charge is calculated in detail for a magnet model consisting of two interacting point charges which are constrained to move in a circular orbit on a frictionless ring with a compensating negative charge at the center.
\end{abstract}




\section{INTRODUCTION}

The interaction of a magnet and an external point charge has attracted attention for over a century. In 1967, Shockley and James[1] pointed out a paradox for this system; there is an apparent momentum imbalance between the charge and the magnet when the magnetic moment of the magnet changes due to some internal forces. Thus it was suggested that one can imagine the magnet to consist of two counter-rotating disks of opposite charge; the disks are brought into frictional contact and come to rest so that the magnetic moment is reduced to zero. The Faraday induction field arising from the changing magnetic moment gives an obvious electric force to change the linear momentum of the external charge; however, the balancing change in the linear momentum of the magnet is not so obvious. This "Shockley-James paradox" attracted the attention of Coleman and Van Vleck[2] who provided a sophisticated discussion of the situation and directed attention to the relativistic mechanical momentum of the charge carriers for the magnetic moment. The relativistic "hidden mechanical momentum" emphasized by Coleman and Van Vleck has been widely adopted. In his "Resource Letter EM-1:Electromagnetic Momentum," Griffiths lists twentythree articles on "hidden momentum." [3] "Hidden mechanical momentum" now appears in the electromagnetism textbooks. [4] [5] It has been used as the basis for erroneous descriptions of the equation of motion of a magnetic moment in an electric field, [6] and of the classical electromagnetic aspects of the Aharonov-Casher phase shift [7] and the AharonovBohm phase shift, [8]. Most recently the ideas of "hidden mechanical momentum" were used in connection with rebuttals[9] to Mansuripur's claim[10] of the inconsistency of the Lorentz force law with special relativity.

In the present article, we point out that Coleman and Van Vleck's "hidden-mechanicalmomentum"-solution to the Shockley-James paradox represents a major blunder. The blunder consists in the claim that the "mechanical momentum" of the current carriers of the multiparticle magnet provides the internal momentum in the magnet (required by Lorentz invariance) which balances the familiar electromagnetic momentum arising from the electric field of the external charge and magnetic field of the magnet.

Indeed, the claim of importance for "mechanical momentum" in a multiparticle electromagnetic system should immediately be regarded with suspicion. For a multiparticle electromagnetic system in a situation where particle collisions are not important, it seems 
highly unlikely that the mass of the current carriers is of significance compared to the electromagnetic interactions between the charges. Thus, for example, the textbook discussions of the self-inductance of a circuit make no reference whatsoever to the mass of the charges carrying the currents of the circuit. Rather it is the interaction of the accelerating charges through their electromagnetic fields which overwhelms any mechanical inertia of the charges' mass, and so determines the acceleration of the charges.[11]

In the present article, we point out that in the interacting-multiparticle electromagnetic situation, the external charge induces a crucial electrostatic polarization of the magnet which leads to an internal electromagnetic momentum in the magnet where both the electric and magnetic fields for the momentum are contributed by the magnet particles. This internal electromagnetic momentum for the interacting multiparticle situation is equal in magnitude and opposite in direction compared to the familiar external electromagnetic field momentum where the electric field is contributed by the external charged particle and the magnetic field is that due to the magnet. As a transparent illustration of these ideas, we discuss a situation corresponding in essence to the Shockley-James paradox. In the present article, for reasons of clarity, external forces (rather than internal frictional forces) are applied which give the magnet a changing magnetic moment, and therefore an electromagnetic impulse is delivered to the external charge. Momentum conservation is exhibited in detail for a magnet model consisting of two moving and interacting point charges of low velocity which are constrained to move in a circular orbit on a frictionless ring with a balancing negative charge at the center. In this easily-calculated example, the magnet contains internal electromagnetic momentum, the total electromagnetic momentum of the system vanishes, and the canonical momentum of the external charge remains constant (as was noted by Coleman and Van Vleck). The correct understanding of the magnet-point charge interaction has major implications for our understanding of the motion of a magnetic moment, and of the experimentally observed Aharonov-Bohm and Aharonov-Casher phase shifts. 


\section{USE OF THE DARWIN LAGRANGIAN APPROXIMATION}

\section{A. The Darwin Lagrangian}

Except for the blunder in connection with the final step of their analysis, Coleman and Van Vleck provide an extremely helpful discussion of the Shockley-James paradox. Here we will follow major portions of their discussion. Coleman and Van Vleck note that the Shockley-James paradox must be treated through order $1 / c^{2}$ (in gaussian units) in the electromagnetic interactions since the obvious Faraday induction field acting on the external charge is of order $1 / c^{2}$. To this $1 / c^{2}$-order, the forces between a magnet and a point charge can be understood in terms of the electromagnetic interactions described by the Darwin Lagrangian. This truncated description of electromagnetism includes all electromagnetic forces through order $1 / c^{2}$ but omits radiation fields entirely. The Darwin Lagrangian for point charges $e_{i}$ with masses $m_{i}$, displacements $\mathbf{r}_{i}$, and velocities $\mathbf{v}_{i}$ takes the form[12]

$$
\begin{aligned}
\mathcal{L}= & \sum_{i=1}^{i=N} m_{i} c^{2}\left(-1+\frac{\mathbf{v}_{i}^{2}}{2 c^{2}}+\frac{\left(\mathbf{v}_{i}^{2}\right)^{2}}{8 c^{4}}\right)-\frac{1}{2} \sum_{i=1}^{i=N} \sum_{j \neq i} \frac{e_{i} e_{j}}{\left|\mathbf{r}_{i}-\mathbf{r}_{j}\right|} \\
& +\frac{1}{2} \sum_{i=1}^{i=N} \sum_{j \neq i} \frac{e_{i} e_{j}}{2 c^{2}}\left[\frac{\mathbf{v}_{i} \cdot \mathbf{v}_{j}}{\left|\mathbf{r}_{i}-\mathbf{r}_{j}\right|}+\frac{\mathbf{v}_{i} \cdot\left(\mathbf{r}_{i}-\mathbf{r}_{j}\right) \mathbf{v}_{j} \cdot\left(\mathbf{r}_{i}-\mathbf{r}_{j}\right)}{\left|\mathbf{r}_{i}-\mathbf{r}_{j}\right|^{3}}\right]
\end{aligned}
$$

\section{B. Equations of Motion}

The Lagrangian equations of motion derived from Eq. (1) can be rewritten in Newtonian form with the force given by the Lorentz force due to the other charges

$$
\begin{aligned}
& \frac{d \mathbf{p}_{i}^{\text {mechanical }}}{d t}=\frac{d}{d t}\left[m_{i} \gamma_{i} \mathbf{v}_{i}\right]=\frac{d}{d t}\left[\frac{m_{i} \mathbf{v}_{i}}{\left(1-\mathbf{v}_{i}^{2} / c^{2}\right)^{1 / 2}}\right] \approx \frac{d}{d t}\left[m_{i}\left(1+\frac{\mathbf{v}_{i}^{2}}{2 c^{2}}\right) \mathbf{v}_{i}\right] \\
= & e_{i} \sum_{j \neq i} \mathbf{E}_{j}\left(\mathbf{r}_{i}, t\right)+e_{i} \frac{\mathbf{v}_{i}}{c} \times \sum_{j \neq i} \mathbf{B}_{j}\left(\mathbf{r}_{i}, t\right)
\end{aligned}
$$

The electric field due to the $j$ th particle is given through order $1 / c^{2}$ by 13$]$

$$
\begin{aligned}
\mathbf{E}_{j}(\mathbf{r}, \mathbf{t})= & e_{j} \frac{\left(\mathbf{r}-\mathbf{r}_{j}\right)}{\left|\mathbf{r}-\mathbf{r}_{j}\right|^{3}}\left[1+\frac{\mathbf{v}_{j}^{2}}{2 c^{2}}-\frac{3}{2}\left(\frac{\mathbf{v}_{j} \cdot\left(\mathbf{r}-\mathbf{r}_{j}\right)}{c\left|\mathbf{r}-\mathbf{r}_{j}\right|}\right)^{2}\right] \\
& -\frac{e_{j}}{2 c^{2}}\left(\frac{\mathbf{a}_{j}}{\left|\mathbf{r}-\mathbf{r}_{j}\right|}+\frac{\mathbf{a}_{j} \cdot\left(\mathbf{r}-\mathbf{r}_{j}\right)\left(\mathbf{r}-\mathbf{r}_{j}\right)}{\left|\mathbf{r}-\mathbf{r}_{j}\right|^{3}}\right)
\end{aligned}
$$

and the magnetic field is

$$
\mathbf{B}_{j}(\mathbf{r}, t)=e_{j} \frac{\mathbf{v}_{j}}{c} \times \frac{\left(\mathbf{r}-\mathbf{r}_{j}\right)}{\left|\mathbf{r}-\mathbf{r}_{j}\right|^{3}}
$$


where the quantity $\mathbf{a}_{j}$ in Eq. (3) corresponds to the acceleration of the $j$ th particle.

\section{Canonical Linear Momentum from the Darwin Lagrangian}

Following from the Darwin Lagrangian in Eq. (11), we find the canonical linear momentum of the $i$ th charge

$$
\frac{\partial \mathcal{L}}{\partial \mathbf{v}_{i}}=\mathbf{p}_{i}^{\text {canonical }}=m_{i}\left(1+\frac{\mathbf{v}_{i}^{2}}{2 c^{2}}\right) \mathbf{v}_{i}+\sum_{j \neq i} \frac{e_{i} e_{j}}{2 c^{2}}\left(\frac{\mathbf{v}_{j}}{\left|\mathbf{r}_{i}-\mathbf{r}_{j}\right|}+\frac{\mathbf{v}_{j} \cdot\left(\mathbf{r}_{i}-\mathbf{r}_{j}\right)\left(\mathbf{r}_{i}-\mathbf{r}_{j}\right)}{\left|\mathbf{r}_{i}-\mathbf{r}_{j}\right|^{3}}\right)
$$

The canonical linear momentum includes a mechanical linear momentum of the $i$ th particle

$$
\mathbf{p}_{i}^{\text {mechanical }}=m_{i} \gamma_{i} \mathbf{v}_{i} \approx m_{i}\left[1+v_{i}^{2} /\left(2 c^{2}\right)\right] \mathbf{v}_{i}=m_{i} \mathbf{v}_{i}+m_{i} v_{i}^{2} \mathbf{v}_{i} /\left(2 c^{2}\right)
$$

and also the electromagnetic linear momenta $\mathbf{p}_{i}^{e m}$ associated with the electric field $\mathbf{E}_{i}$ of the $i$ th particle and magnetic fields $\mathbf{B}_{j}, j \neq i$, of all the other particles

$$
\mathbf{p}_{i}^{e m}=\sum_{j \neq i} \frac{1}{4 \pi c} \int d^{3} r \mathbf{E}_{i} \times \mathbf{B}_{j}=\sum_{j \neq i} \frac{e_{i} e_{j}}{2 c^{2}}\left(\frac{\mathbf{v}_{j}}{\left|\mathbf{r}_{i}-\mathbf{r}_{j}\right|}+\frac{\mathbf{v}_{j} \cdot\left(\mathbf{r}_{i}-\mathbf{r}_{j}\right)\left(\mathbf{r}_{i}-\mathbf{r}_{j}\right)}{\left|\mathbf{r}_{i}-\mathbf{r}_{j}\right|^{3}}\right)
$$

\section{VANISHING TOTAL MOMENTUM FOR A POINT CHARGE AT REST OUTSIDE A MAGNET}

\section{A. Vanishing Total Momentum from the Requirement of Lorentz Invariance}

Coleman and Van Vleck provide a sophisticated proof (based on Lorentz invariance) that the total momentum of a magnet-point charge system must vanish when held at steady state. In the present article, we are interested in providing physical insight into the aspect that Coleman and Van Vleck missed. Thus, rather than providing a general discussion, we will introduce a simple model for the magnet which we will use later in explicit calculations.

We consider the situation of a point charge $q$ at rest outside a multiparticle magnet which is modeled as $N$ point charges $e_{i}, i=1,2, \ldots N$, of equal charge $e$ and mass $m$, moving without friction with a steady current in a circle of radius $R$ about the origin in the $x y$-plane. There is a negative point particle of charge $-N e$ at the center of this circle. Thus the magnetic may be pictured as a set of beads of equal positive charge $e$ sliding on a frictionless ring with a balancing negative charge at the center of the ring. The external point charge $q$ will cause an electrostatic polarization of the magnet so that the spacing between the charges $e_{i}$ will 
vary around the circle. In the interacting-multiparticle case, the masses of the charges $e$ will be of no significance, and the charges will move through the electrostatic charge distribution produced by the external charge $q$. There is an external force of constraint applied to the charge $q$ which keeps $q$ at rest despite the electrostatic attraction. This electrostatic force must be along the line $\mathbf{r}_{q}$ running from the center of the magnet to the charge $q$. There are also radial forces of constraint applied to the magnet charges so that they remain in the circular orbit of radius $R$. The center-of-energy conservation law for a relativistic theory then requires[14]

$$
\sum_{i=1}^{N}\left(\mathbf{F}_{\text {ext } i} \cdot \mathbf{v}_{i}\right) \mathbf{r}_{i}=\frac{d}{d t}\left(U \vec{X}_{C E}\right)-c^{2} \mathbf{P}_{\text {system }}
$$

so that the power delivered by the external forces $\mathbf{F}_{\text {ext } i}$ weighted by the location $\mathbf{r}_{i}$ of the power delivery equals the time-rate-of-change of the system energy $U$ times the center-ofenergy $\vec{X}_{C E}$ minus $c^{2}$ times the total system momentum $\mathbf{P}_{\text {system. }}$ For a real magnet, there are no localized distributed sources of power, and indeed, in our model, there is no power being introduced by the radial forces of constraint; the moving particles of the magnet move perpendicular to the forces of constraint for the circular orbit. In steady state, the energy times the center of energy is not changing in time, and therefore it follows from Eq. (8) that

$$
\mathbf{P}_{\text {system }}=0
$$

the total system momentum (of the magnet and the external charge) must vanish.

\section{B. Familiar External Electromagnetic Momentum}

Now it is a familiar textbook observation that a steady localized current in the presence of an external electric field leads to linear momentum in the electromagnetic field.[15] In the present case, this electromagnetic field momentum $\mathbf{P}_{q-\mu}^{e m}$ involves the electric field $\mathbf{E}_{q}$ of the external charge $q$ and the magnetic field $\mathbf{B}_{\mu}$ of the moving charges of the magnet

$$
\mathbf{P}_{q-\mu}^{e m}=\frac{1}{4 \pi c} \int d^{3} r \mathbf{E}_{q} \times \mathbf{B}_{\mu}=\sum_{i=1}^{N} \frac{q e}{2 c^{2}}\left(\frac{\mathbf{v}_{i}}{\left|\mathbf{r}_{i}-\mathbf{r}_{q}\right|}+\frac{\mathbf{v}_{i} \cdot\left(\mathbf{r}_{i}-\mathbf{r}_{q}\right)\left(\mathbf{r}_{i}-\mathbf{r}_{q}\right)}{\left|\mathbf{r}_{i}-\mathbf{r}_{q}\right|^{3}}\right)
$$


If the charge $q$ at displacement $\mathbf{r}_{q}$ is far from the magnet of radius $R, R<<r_{q}$, then we can expand this expression in powers of $R / r_{q}$. This momentum in Eq. (10) then becomes

$$
\begin{aligned}
\mathbf{P}_{q-\mu}^{e m} \approx & \sum_{i=1}^{N} \frac{q e}{2 c^{2}}\left[\frac{\mathbf{v}_{i}}{r_{q}}\left(1+\frac{\mathbf{r}_{i} \cdot \mathbf{r}_{q}}{r_{q}^{2}}\right)+\frac{\left(\mathbf{v}_{i} \cdot \mathbf{r}_{i}-\mathbf{v}_{i} \cdot \mathbf{r}_{q}\right)\left(\mathbf{r}_{i}-\mathbf{r}_{q}\right)}{r_{q}^{3}}\left(1+3 \frac{\mathbf{r}_{i} \cdot \mathbf{r}_{q}}{r_{q}^{2}}\right)\right] \\
= & \frac{q e}{2 c^{2}} \sum_{i=1}^{N}\left\{\left(\frac{\mathbf{v}_{i}}{r_{q}}+\frac{\left(\mathbf{v}_{i} \cdot \mathbf{r}_{q}\right) \mathbf{r}_{q}}{r_{q}^{3}}\right)+\left(\frac{-\left(\mathbf{v}_{i} \cdot \mathbf{r}_{i}\right) \mathbf{r}_{q}}{r_{q}^{3}}+\frac{3\left(\mathbf{v}_{i} \cdot \mathbf{r}_{q}\right)\left(\mathbf{r}_{i} \cdot \mathbf{r}_{q}\right) \mathbf{r}_{q}}{r_{q}^{5}}\right)\right. \\
& \left.+\left(\frac{\left(\mathbf{r}_{i} \cdot \mathbf{r}_{q}\right) \mathbf{v}_{i}-\left(\mathbf{v}_{i} \cdot \mathbf{r}_{q}\right) \mathbf{r}_{i}}{r_{q}^{3}}\right)\right\}+O\left(1 / r_{q}^{3}\right) \\
= & \frac{1}{c}\left(\frac{e}{2 c} \sum_{i=1}^{N} \mathbf{r}_{i} \times \mathbf{v}_{i}\right) \times \frac{q \mathbf{r}_{q}}{r_{q}^{3}}=\frac{1}{c} \vec{\mu} \times \frac{q \mathbf{r}_{q}}{r_{q}^{3}}=\frac{-1}{c} \vec{\mu} \times \mathbf{E}_{q}(0)
\end{aligned}
$$

where $\vec{\mu}$ is the magnetic moment of the magnet, $\mathbf{E}_{q}(0)=-q \mathbf{r}_{q} / r_{q}^{2}$, and where we have used $\sum \mathbf{v}_{i}=(d / d t) \sum \mathbf{r}_{i}=0, \sum \mathbf{v}_{i} \cdot \mathbf{r}_{i}=(d / d t) \sum r_{i}^{2} / 2=0$, and $\sum\left(\mathbf{v}_{i} \cdot \mathbf{r}_{q}\right)\left(\mathbf{r}_{i} \cdot \mathbf{r}_{q}\right)=(d / d t) \sum\left(\mathbf{r}_{i}\right.$. $\left.\mathbf{r}_{q}\right)\left(\mathbf{r}_{i} \cdot \mathbf{r}_{q}\right) / 2=0$ for a steady-state magnet current. This result gives the electromagnetic field momentum $\mathbf{P}_{q-\mu}^{e m}$ which clearly must be balanced by some other momentum so as to meet the relativistic restriction we found above in Eq. (9) that the total system momentum vanishes, $\mathbf{P}_{\text {system }}=0$. Coleman and Van Vleck were aware of the single-charge current-loop model of a magnet,[16], and, without carrying out any detailed calculation, they identified the missing piece of the total momentum as mechanical momentum associated with the motion of the masses of the current carriers in the magnet.[17] Such an identification is a blunder for an interacting-multiparticle magnet.

\section{Vanishing Total Electromagnetic Momentum for an Interacting-Multiparticle}

\section{Magnet}

Let us go back and reconsider the standard textbook calculation of the electromagnetic field momentum for a magnet-point charge system.[15] For a localized steady-state situation, the total electromagnetic field momentum is given by

$$
\mathbf{P}_{\text {total }}^{e m}=\frac{1}{4 \pi c} \int d^{3} r \mathbf{E} \times \mathbf{B}=\frac{-1}{4 \pi c} \int d^{3} r \nabla \Phi \times \mathbf{B}=\frac{1}{4 \pi c} \int d^{3} r \Phi \nabla \times \mathbf{B}=\frac{1}{c^{2}} \int d^{3} r \Phi \mathbf{J}
$$

where $\mathbf{E}=-\nabla \Phi$, and we have used the assumption that both the charge and current distributions are localized so as to drop the surface term involved in the partial integration. In the textbook example, it is assumed that the only contribution to the electrostatic potential 
$\Phi$ comes from the external charge $q$,

$$
\Phi(\mathbf{r})=\Phi_{q}(\mathbf{r})=\frac{q}{\left|\mathbf{r}-\mathbf{r}_{q}\right|}
$$

However, for a multiparticle magnet, the speed of the charges will become ever smaller for a fixed magnetic moment as the number of charges is increased. Then we expect that the external charge $q$ will cause a polarization of the magnet which is approximately that of electrostatics. Now if the charge $q$ produces an electrostatic polarization of the magnet, then there is a second source of the electrostatic potential, that due to the electrostatic charge distribution of the magnet. Therefore instead of Eq. (13), we must write

$$
\Phi(\mathbf{r})=\frac{q}{\left|\mathbf{r}-\mathbf{r}_{q}\right|}+\left(\sum_{i=1}^{N} \frac{e}{\left|\mathbf{r}-\mathbf{r}_{i}\right|}+\frac{-N e}{|\mathbf{r}|}\right)
$$

The first term on the right-hand side is the electrostatic potential $\Phi_{q}(\mathbf{r})$ of the external charge $q$, and the second term in the round brackets includes the electrostatic potential of the moving magnet charges $e_{i}$ together with the potential of the central negative charge. Now the only current density $\mathbf{J}$ is that associated with the moving charges of the magnet

$$
\mathbf{J}(\mathbf{r}, t)=\sum_{i=1}^{N} e \mathbf{v}_{i} \delta^{3}\left[\mathbf{r}-\mathbf{r}_{i}(t)\right]
$$

Thus the total electromagnetic field momentum in Eq. (12) is given by

$$
\mathbf{P}_{\text {total }}^{e m}=\frac{1}{c^{2}} \sum_{i=1}^{N} e \mathbf{v}_{i}(t) \Phi\left(\mathbf{r}_{i}, t\right)=\frac{1}{c^{2}} \sum_{i=1}^{N} e \mathbf{v}_{i}(t) \text { const }=0
$$

Here we have used two facts. First, in electrostatic equilibrium for the multiparticle situation, the tangential component of the electric field at each particle vanishes so that the electrostatic potential along the magnet is a constant. Second, in steady state, the current is constant, so that $\sum e \mathbf{v}_{i}=0$.

Thus we find the situation that the total electromagnetic momentum of the entire system vanishes $\mathbf{P}_{\text {total }}^{e m}=0$ because the electromagnetic field momentum $\mathbf{P}_{q-\mu}^{e m}$ (arising from the electric field of the charge $q$ and magnetic field of the magnet) is balanced by the electromagnetic momentum $\mathbf{P}_{\mu}^{e m}$ (arising from the induced electrostatic charge distribution of the magnet and the magnetic fields of the charges of the magnet). This second electromagnetic momentum $\mathbf{P}_{\boldsymbol{\mu}}^{e m}$ involves only charges of the magnet and can be regarded as momentum internal to the magnet. 
In an earlier article, the internal electromagnetic momentum was calculated and exhibited explicitly for the simple case of a two-moving-particle magnet. [18] This two-particle magnet was treated for moving charges at finite velocity (retaining the squares of the velocity), and therefore both internal mechanical momentum and internal electromagnetic momentum are present in the magnet and contribute to the total momentum required by relativity in Eq. (9). It is only in the multiparticle case or the low-velocity few-particle case that the internal electromagnetic momentum is dominant and that the internal mechanical momentum is negligible. It is only in the multiparticle case or the low-velocity few-particle case that the induced charge distribution for the magnet goes over to the electrostatic distribution and the total electromagnetic momentum vanishes.

\section{Remarks on Electromagnetic Momentum in the Physics Literature}

The evaluation of the electromagnetic field momentum for a magnet-point charge system in both the textbook and research literature usually makes the assumption (often not stated explicitly) that the currents of the magnet are held at their unperturbed values despite the presence of the external charge.[19] Such unperturbed currents require that the external forces (which hold the currents to their unperturbed values) deliver energy locally at one part of the current distribution and remove energy locally at a different part of the current distribution so that there is a net flow of power and an associated net momentum density.[20] [21] [22] Such local, power-delivering external forces do not occur in nature.

It has been remarked that if the magnet is shielded by a closed conductor, then the total electromagnetic field momentum will vanish since the external electric field of the charge $q$ would be screened out of the region of the currents. [20] 21] However, this has been regarded as a special situation. We suggest that the vanishing of the total electromagnetic

field momentum is the general situation which holds in all cases involving an interactingmultiparticle magnet which has no local power-delivering external forces. 


\section{ELECTROMAGNETIC MOMENTUM FOR A TWO-MOVING- INTERACTING-PARTICLE MAGNET}

\section{A. The Two-Moving-Interacting-Particle Model for a Magnet}

In order to emphasize the vanishing total electromagnetic momentum for the situation of a multiparticle magnet and a point charge, we will carry out the explicit calculation for a two-moving-interacting-particle magnet following the model mentioned above. Since this is a few-particle magnet, we must work in the low-velocity limit where we ignore terms in $v_{i}^{2}$, the squares of the velocities of the moving charges in the magnet.

We consider two charges $e_{i}, i=1,2$, of equal charge $e$ located at

$$
\mathbf{r}_{i}=R\left(\widehat{i} \cos \phi_{i}+\widehat{j} \sin \phi_{i}\right)=R \widehat{r}_{i}
$$

in the $x y$-plane, which are free to move on a frictionless circular ring of radius $R$ about the coordinate origin with a negative charge $-2 e$ in the center. When there is no external charge $q$ present, the steady-state motion of the charges is given by unperturbed values,

$$
\phi_{0 i}=\omega_{0} t+i \pi+\theta_{0}, \quad i=1,2
$$

where $\theta_{0}$ is an initial phase. In our calculations, we will average over the initial phase $\theta_{0}$. The tangential direction $\widehat{\phi}_{i}$ for the $i$ th particle is given by

$$
\widehat{\phi}_{i}=-\widehat{i} \sin \phi_{i}+\widehat{j} \cos \phi_{i}
$$

\section{B. Perturbation of the Particle Motion Due to the External Charge $q$}

The external charge $q$ is located at $\mathbf{r}_{q}$ in the $x y$-plane outside the magnet, $R<<r_{q}$,

$$
\mathbf{r}_{q}=r_{q}\left(\widehat{i} \cos \phi_{q}+\widehat{j} \sin \phi_{q}\right)=r_{q} \widehat{r}_{q}
$$

and

$$
\widehat{\phi}_{q}=\left(-\hat{i} \sin \phi_{q}+\widehat{j} \cos \phi_{q}\right)
$$

We assume that the two charges are are moving slowly so that they are nearly in electrostatic equilibrium, with the average angle, $\left(\phi_{1}+\phi_{2}\right) / 2=\omega_{0} t+$ const, moving at constant angular 
velocity $\omega_{0}$. Due to the presence of the external charge $q$, the charges are displaced from the unperturbed angular positions $\phi_{0 i}$ over to

$$
\phi_{i}=\phi_{0 i}+\eta_{i}
$$

so that $\mathbf{r}_{i}=\widehat{r}_{i} R=\widehat{i} R \cos \left(\phi_{0 i}+\eta_{i}\right)+\widehat{j} R \sin \left(\phi_{0 i}+\eta_{i}\right)$. Through first order in the perturbation caused by the charge $q$, we find

$\widehat{r}_{i}=\widehat{i} \cos \left(\phi_{0 i}+\eta_{i}\right)+\widehat{j} \sin \left(\phi_{0 i}+\eta_{i}\right) \approx \widehat{i}\left[\cos \phi_{0 i}-\eta_{i} \sin \phi_{0 i}\right]+\widehat{j}\left[\sin \phi_{0 i}+\eta_{i} \cos \phi_{0 i}\right]=\widehat{r}_{0 i}+\widehat{\phi}_{0 i} \eta_{i}$.

using the first-order approximations $\sin \eta_{i} \approx \eta_{i}$ and $\cos \eta_{i} \approx 1$. We will also need the velocity $\mathbf{v}_{i}$ of the $i$ th particle, given by

$$
\mathbf{v}_{i}=\frac{d \mathbf{r}_{i}}{d t}=\widehat{\phi}_{i} v_{i}=R\left(\omega_{0}+\frac{d \eta_{i}}{d t}\right)\left[-\widehat{i} \sin \left(\phi_{0 i}+\eta_{i}\right)+\widehat{j} \cos \left(\phi_{0 i}+\eta_{i}\right)\right]
$$

The unit vector $\widehat{\phi}_{i}$ in the direction of the velocity can be approximated through first order in the perturbation as

$$
\begin{aligned}
\widehat{\phi}_{i} & =-\widehat{i} \sin \left(\phi_{0 i}+\eta_{i}\right)+\widehat{j} \cos \left(\phi_{0 i}+\eta_{i}\right) \approx-\widehat{i}\left[\sin \phi_{0 i}+\eta_{i} \cos \phi_{0 i}\right]+\widehat{j}\left[\cos \phi_{0 i}-\eta_{i} \sin \phi_{0 i}\right] \\
& =\widehat{\phi}_{0 i}-\widehat{r}_{0 i} \eta_{i}
\end{aligned}
$$

and the particle velocity is approximated as

$$
\mathbf{v}_{i}=R\left(\omega_{0}+\frac{d \eta_{i}}{d t}\right) \widehat{\phi}_{0 i}-\omega_{0} R \eta_{i} \widehat{r}_{0 i}
$$

where $\omega_{0} R=v_{0}$ is the speed of the unperturbed charges.

When the unperturbed angular velocity $\omega_{0}$ is very small, we may use the nonrelativistic form of Newton's second law for the motion of the charged particles and use the electrostatic fields for the forces. Thus the tangential acceleration of the $i$ th particle is given by

$$
\begin{aligned}
m R \frac{d^{2} \phi_{i}}{d t^{2}} & =m R \frac{d^{2} \eta_{i}}{d t^{2}}=\widehat{\phi}_{i} \cdot\left[e \mathbf{E}_{q}\left(\mathbf{r}_{i}\right)+e \mathbf{E}_{j \neq i}\left(\mathbf{r}_{i}\right)\right]=\widehat{\phi}_{i} \cdot\left(\frac{-e q \widehat{r}_{q}}{r_{q}^{2}}+\frac{e^{2}\left(\mathbf{r}_{i}-\mathbf{r}_{j \neq i}\right)}{\left|\mathbf{r}_{i}-\mathbf{r}_{j \neq i}\right|^{3}}\right) \\
& =e\left(-q \frac{\widehat{\phi}_{i} \cdot \widehat{r}_{q}}{r_{q}^{2}}-e \frac{\widehat{\phi}_{i} \cdot \widehat{r}_{j \neq i} R}{\left|\mathbf{r}_{i}-\mathbf{r}_{j \neq i}\right|^{3}}\right) \approx e\left(-q \frac{\widehat{\phi}_{0 i} \cdot \widehat{r}_{q}}{r_{q}^{2}}-e \frac{\left(\eta_{i}-\eta_{j \neq i}\right) R}{(2 R)^{3}}\right)
\end{aligned}
$$

through first order in the perturbation, where we have used $\widehat{\phi}_{i} \cdot \widehat{r}_{i}=0$ and

$$
\begin{aligned}
\widehat{\phi}_{i} \cdot \widehat{r}_{j \neq i} & \left.=\left[-\widehat{i} \sin \left(\phi_{0 i}+\eta_{i}\right)+\widehat{j} \cos \left(\phi_{0 i}+\eta_{i}\right)\right] \cdot \widehat{i} \cos \left(\phi_{0 j \neq i}+\eta_{j \neq i}\right)+\widehat{j} \sin \left(\phi_{0 j \neq i}+\eta_{j \neq i}\right)\right] \\
& =\sin \left(\phi_{0 j \neq i}+\eta_{j \neq i}-\phi_{0 i}-\eta_{i}\right)=\sin \left(\eta_{i}-\eta_{j \neq i}\right) \approx \eta_{i}-\eta_{j \neq i}
\end{aligned}
$$


since $\phi_{01}-\phi_{02}=\pi$. The equation (26) is odd under the interchange of the two particles. Then for the steady state situation, we must have $\eta_{1}=-\eta_{2}$, and so equation (26) becomes

$$
m R \frac{d^{2} \eta_{i}}{d t^{2}}=e\left(-q \frac{\widehat{\phi}_{0 i} \cdot \widehat{r}_{q}}{r_{q}^{2}}-e \frac{2 \eta_{i} R}{(2 R)^{3}}\right)
$$

Then from the unperturbed motions $\phi_{0 i}=\omega_{0} t+i \pi+\theta_{0}$, we find the steady-state angular perturbation given by

$$
\eta_{i}=e \frac{-q}{r_{q}^{2}} \widehat{r}_{q} \cdot \widehat{\phi}_{0 i} \frac{1}{\left[-m R \omega_{0}^{2}+e^{2} /(2 R)^{2}\right]}
$$

Now here we are interested only in the low-velocity limit, which will correspond to multiparticle behavior where the electric charge distribution is essentially the electrostatic distribution. Therefore we will retain terms only through first order in the particle speed $v_{0 i}=R \omega_{0}$. Accordingly, for $m R^{2} \omega_{0}^{2}<<e^{2} / R$, the angular perturbation in Eq. (29) becomes

$$
\eta_{i}=e \frac{-q}{r_{q}^{2}} \widehat{r}_{q} \cdot \widehat{\phi}_{0 i} \frac{1}{\left[e^{2} /(2 R)^{2}\right]}=\frac{-4 q R^{2}}{e r_{q}^{2}} \widehat{r}_{q} \cdot \widehat{\phi}_{0 i}=\frac{4 q R^{2}}{e r_{q}^{2}} \sin \left(\phi_{0 i}-\phi_{q}\right)
$$

which is the same as the electrostatic perturbation. Taking the time derivative in Eq. (30) and noting $d \widehat{\phi}_{0 i} / d t=-\omega_{0} \widehat{r}_{0 i}$, we have

$$
\frac{d \eta_{i}}{d t}=\frac{4 q \omega_{0} R^{2}}{e r_{q}^{2}} \widehat{r}_{q} \cdot \widehat{r}_{0 i}=\frac{4 q \omega_{0} R^{2}}{e r_{q}^{2}} \cos \left(\phi_{0 i}-\phi_{q}\right)
$$

\section{Vanishing Total Electromagnetic Linear Momentum}

When we average over the initial angle $\theta_{0}$ and carry out our calculation in the low-velocity approximation where we ignore terms in $v_{i}^{2}$, then the two-particle magnet behaves as though the circle of orbital motion is an equipotential and hence the total electromagnetic momentum of the system vanishes. The familiar external electromagnetic momentum is that given in Eq. (11). The internal electromagnetic linear momentum is that arising from electric and magnetic fields of the charges of the magnet alone. Thus here the internal electromagnetic momentum $\mathbf{P}_{\mu}^{e m}$ of the magnet is given (through first order in the perturbation) by

$$
\begin{aligned}
\mathbf{P}_{\mu}^{e m} & =\sum_{i=1}^{2} \int d^{3} r \frac{1}{4 \pi c} \mathbf{E}_{i} \times \mathbf{B}_{j \neq i}=\sum_{i=1}^{2} \frac{e^{2}}{2 c^{2}}\left(\frac{\mathbf{v}_{j \neq i}}{\left|\mathbf{r}_{i}-\mathbf{r}_{j \neq i}\right|}+\frac{\mathbf{v}_{j \neq i} \cdot\left(\mathbf{r}_{i}-\mathbf{r}_{j \neq i}\right)\left(\mathbf{r}_{i}-\mathbf{r}_{j \neq i}\right)}{\left|\mathbf{r}_{i}-\mathbf{r}_{j \neq i}\right|^{3}}\right) \\
& \approx \sum_{i=1}^{2} \frac{e^{2}}{2 c^{2}}\left(\frac{\mathbf{v}_{j \neq i}}{2 R}+\frac{\left(v_{j \neq i} \widehat{\phi}_{j \neq i} \cdot \widehat{r}_{i}\right)\left(\widehat{r}_{i}-\widehat{r}_{j \neq i}\right) R^{2}}{(2 R)^{3}}\right)
\end{aligned}
$$


Now using Eq. (27), this becomes through first order in the perturbation

$$
\begin{aligned}
\mathbf{P}_{\mu}^{e m} & \approx \sum_{i=1}^{2} \frac{e^{2}}{2 c^{2}}\left(\frac{\mathbf{v}_{j \neq i}}{2 R}+\frac{v_{j \neq i}\left(\eta_{j \neq i}-\eta_{i}\right)\left(\widehat{r}_{i}-\widehat{r}_{j \neq i}\right) R^{2}}{(2 R)^{3}}\right) \\
& \approx \sum_{i=1}^{2} \frac{e^{2}}{2 c^{2}}\left(\frac{\mathbf{v}_{j \neq i}}{2 R}+\frac{v_{0}\left(-2 \eta_{i}\right)\left(2 \widehat{r}_{0 i}\right) R^{2}}{(2 R)^{3}}\right)
\end{aligned}
$$

Now averaging over the initial angle $\theta_{0}$, we note that $\left\langle\sin \theta_{0}\right\rangle=\left\langle\cos \theta_{0}\right\rangle=\left\langle\sin \theta_{0} \cos \theta_{0}\right\rangle=0$, while $\left\langle\sin ^{2} \theta_{0}\right\rangle=\left\langle\cos ^{2} \theta_{0}\right\rangle=1 / 2$. From Eq. (25) we have $\left\langle\mathbf{v}_{i}\right\rangle=\left\langle\mathbf{v}_{j \neq i}\right\rangle=0$. Also, $\left\langle\eta_{i} \widehat{r}_{0 i}\right\rangle=\widehat{\phi}_{q} 2 q R^{2} /\left(e r_{q}^{2}\right)$. Therefore Eq. (33) becomes

$$
\begin{aligned}
\left\langle\mathbf{P}_{\mu}^{e m}\right\rangle & \approx \sum_{i=1}^{2} \frac{e^{2}}{2 c^{2}}\left(\frac{\left\langle\mathbf{v}_{j \neq i}\right\rangle}{2 R}-\frac{v_{0}\left\langle\eta_{i} \widehat{r}_{0 i}\right\rangle}{2 R}\right) \\
& =\sum_{i=1}^{2} \frac{e^{2} v_{0}}{2 c^{2}}\left(\frac{4 q R^{2}}{e r_{q}^{2}}\right)\left[\frac{0}{2 R}-\frac{\widehat{\phi}_{q} / 2}{2 R}\right]=2 \frac{-e v_{0} R}{2 c^{2}}\left(\frac{q}{r_{q}^{2}}\right) \widehat{\phi}_{q} \\
& =\frac{1}{c}\left(\frac{e}{2 c} \sum_{i=1}^{2} \mathbf{r}_{i} \times \mathbf{v}_{i}\right) \times \frac{-q \mathbf{r}_{q}}{r_{q}^{3}}=\frac{1}{c} \vec{\mu} \times \mathbf{E}(0)
\end{aligned}
$$

where the magnetic moment of our two-moving-interacting-particle model is

$$
\vec{\mu}=\frac{e}{2 c} \sum_{i=1}^{2} \mathbf{r}_{i} \times \mathbf{v}_{i}=\widehat{k} 2 \frac{e}{2 c} v_{0} R=\widehat{k} 2 \frac{e}{2 c} R^{2} \omega_{0}
$$

and where $\mathbf{E}_{q}(0)=-q \mathbf{r}_{q} / r_{q}^{3}$ is the electric field of the external charge $q$ at the position of the magnet. We see that for this specific example, the internal electromagnetic linear momentum is indeed just the negative of the familiar external electromagnetic momentum (due to the electric field of the external charge and the magnetic field of the magnet) which was calculated above in Eq. (11). Thus in this low-velocity case for the magnet charges where the center of energy of the system of magnet and point charge is at rest, the total electromagnetic momentum (internal plus external) vanishes.

\section{LINEAR MOMENTUM CONSERVATION AND THE SHOCKLEY-JAMES PARADOX}

\section{A. Momentum Balance for a Magnet and a Point Charge When the Magnetic}

\section{Moment Changes}

At this point, we will consider the momentum balance for a magnet and a point charge when the magnetic moment of the magnet is changing. This is the situation of the ShockleyJames paradox. The description of a magnet in terms of rotating charged disks, which was 
referred to in the Introduction and which appears in the discussion of Shockley and James[1] and of Coleman and Van Vleck, [2] seems dangerously inexact for a relativistic analysis. Thus rather than deal with the ambiguous internal forces mentioned by Shockley and James, we will discuss an equivalent situation where well-defined external forces provide the change in the magnetic moment of the magnet. If one wishes to make the situation closer to that involving frictional forces between two counter-rotating oppositely-charge disks, one need only reverse the sign of the charge carriers in the model as well as the direction of rotation and of the applied external forces, and then average over the two oppositely-charged-magnet models.

Specifically, we will consider a charge $q$ outside a magnet, the magnet being modeled as above as a set of charges $e_{i}$ which are free to move on a frictionless circular ring with a charge-compensating negative charge $-N e$ at the center of the ring . Next tangential external forces are applied to the charges of the magnet so as to change the magnetic moment. These external forces accelerate the magnet charges $e_{i}$ causing a changing magnetic moment. The changing magnetic moment causes an induced electric field back at the position of the external charge $q$. The electric field delivers an impulse to the charge $q$. But how do we balance momentum in this situation? The paradox arises from the obvious transfer of linear momentum to the external charge without an obvious linear impulse being provided by the external forces.

The naive discussion, which suggests a paradox, implicitly assumes that the charges of the arrangement are uniformly spaced around the ring. Indeed, if the charges of the magnet were uniformly spaced around the circle and the applied tangential forces were of the same magnitude, then there would be no net linear momentum introduced by the external forces while the Faraday induction field due to the changing magnet moment indeed delivers a net impulse to the external charge which is balanced by an increase in the external electromagnetic momentum associated with the constant canonical momentum of the external charge. What is lacking in this analysis is the recognition that if there is an electrostatic polarization of the magnet (caused by the external charge $q$ ), then the charges of the arrangement are not uniformly space around the circular orbit, and therefore there can be a net force on the magnet due to the external forces and also a change in the internal momentum of the magnet.

We have seen that an interacting-multiparticle magnet is polarized by an external charge 
and that this polarization leads to vanishing total electromagnetic momentum for the system. In this case, there are no external tangential forces on the charges of the magnet which violate the conditions of steady-state equilibrium. When external forces are applied to change the magnetic moment of the magnet, these external forces can be applied so as to maintain or to violate this steady-state equilibrium. If the forces are applied in a fashion so as to maintain the steady-state equilibrium, then the total electromagnetic momentum remains zero and the external forces produce changes in the nonrelativistic mechanical momentum of the system. We illustrate this situation for our two-particle model.

\section{SHOCKLEY-JAMES SITUATION FOR THE TWO-PARTICLE MAGNET}

\section{A. Changing Magnetic Moment}

We assume that external forces are applied to the charges of the magnet so as to accelerate the charges in such a fashion that the average angular velocity increases linearly in time beginning at time $t=0$, and also in such a fashion that the electrostatic equilibrium situation for small $\omega$ is maintained for $t>0$,

$$
\begin{aligned}
\phi_{i}(t) & =\omega_{0} t+\frac{1}{2} \alpha t^{2}+i \pi+\theta_{0}+\eta_{i} \\
& =\omega_{0} t+\frac{1}{2} \alpha t^{2}+i \pi+\theta_{0}+\frac{4 r q R^{2}}{e r_{q}^{2}} \sin \left(\omega_{0} t+\frac{1}{2} \alpha t^{2}+i \pi+\theta_{0}-\phi_{q}\right)
\end{aligned}
$$

where we have used Eq. (301). Taking the second time derivative of this expression, evaluating it at time $t=0_{+}$, and omitting terms in $\omega_{0}^{2}$, we find

$$
\frac{d^{2} \phi_{i}}{d t^{2}}=\alpha\left[1+\frac{4 q R^{2}}{e r_{q}^{2}} \cos \left(i \pi+\theta_{0}-\phi_{q}\right)\right]=\alpha\left[1+\frac{1}{\omega_{0}} \frac{d \eta_{i}}{d t}\right]
$$

where we have used Eq. (31).

The external forces $\overrightarrow{\mathfrak{F}}(\phi)=\widehat{\phi} \mathfrak{F}(\phi)$ which cause the movable charges of the magnet to accelerate according to Newton's second law must satisfy

$$
\widehat{\phi}_{i} \widehat{\phi}_{i} \cdot \frac{d \mathbf{p}_{i}^{\text {mechanical }}}{d t}=m R \frac{d^{2} \phi_{i}}{d t^{2}} \widehat{\phi}_{i}=\widehat{\phi}_{i} \mathfrak{F}\left(\phi_{i}\right)+e \widehat{\phi}_{i} \widehat{\phi}_{i} \cdot\left(\sum_{j \neq i} \mathbf{E}_{j}\left(\mathbf{r}_{i}\right)+\mathbf{E}_{q}\left(\mathbf{r}_{i}\right)\right)
$$

The electrostatic forces on the charge $e_{i}$ due to the external charge $q$ and due to the other solenoid charges $e_{j \neq i}$ cancel in the direction tangential to the circular orbit and so can 
be ignored. However, the systematic angular acceleration of the magnet charges leads to electromagnetic forces of order $1 / c^{2}$ which are not balanced and can not be ignored. Then Newton's second law gives for the angular acceleration of the charge $e_{i}$

$$
\begin{aligned}
m R \frac{d^{2} \phi_{i}}{d t^{2}} & =\mathfrak{F}_{i}+\widehat{\phi}_{i} \cdot \sum_{j \neq i} \frac{-e^{2}}{2 c^{2}}\left(\frac{\mathbf{a}_{j}}{\left|\mathbf{r}_{i}-\mathbf{r}_{j}\right|}+\frac{\mathbf{a}_{j} \cdot\left(\mathbf{r}_{i}-\mathbf{r}_{j}\right)\left(\mathbf{r}_{i}-\mathbf{r}_{j}\right)}{\left|\mathbf{r}_{i}-\mathbf{r}_{j}\right|^{3}}\right) \\
& =\mathfrak{F}_{i}+\sum_{j \neq i} \frac{-e^{2}}{2 c^{2}} R \frac{d^{2} \phi_{j}}{d t^{2}}\left(\frac{\widehat{\phi}_{i} \cdot \widehat{\phi}_{j}}{\left|\mathbf{r}_{i}-\mathbf{r}_{j}\right|}+\frac{\widehat{\phi}_{j} \cdot\left(\mathbf{r}_{i}\right) \widehat{\phi}_{i} \cdot\left(-\mathbf{r}_{j}\right)}{\left|\mathbf{r}_{i}-\mathbf{r}_{j}\right|^{3}}\right)
\end{aligned}
$$

where $\mathfrak{F}_{i}=\mathfrak{F}\left(\phi_{i}\right)$; here the centripetal acceleration is proportional to $v_{j}^{2}$ and can be ignored in the multiparticle-low-velocity approximation, leaving only the tangential component of acceleration $\mathbf{a}_{j}=\widehat{\phi}_{j} R\left(d^{2} \phi_{j} / d t^{2}\right)$. For the case of a two-particle magnet, $\widehat{\phi}_{0 i} \cdot \widehat{\phi}_{0 j \neq i}=-1$, and $\widehat{\phi}_{j} \cdot\left(\mathbf{r}_{i}\right) \widehat{\phi}_{i} \cdot\left(-\mathbf{r}_{j}\right)=0$ through first order in the perturbation $\eta_{i}$, so that Eq. (39) becomes

$$
m R \frac{d^{2} \phi_{i}}{d t^{2}}=\mathfrak{F}_{i}+e\left[\frac{-e}{2 c^{2}}\left(\frac{-1}{2 R}\right) R \frac{d^{2} \phi_{j \neq i}}{d t^{2}}\right]
$$

Using Eqs. (37) and (40), this requires that the external force $\mathfrak{F}_{i}$ on the $i$ th particle is

$$
\mathfrak{F}_{i}=m R \alpha\left[1+\frac{1}{\omega_{0}} \frac{d \eta_{i}}{d t}\right]-\frac{e^{2}}{4 c^{2}} \alpha\left[1+\frac{1}{\omega_{0}} \frac{d \eta_{j \neq i}}{d t}\right]
$$

The changing magnetic moment for our two-particle magnet is

$$
\frac{d \vec{\mu}}{d t}=\widehat{k} \sum_{i=1}^{2} \frac{e R^{2}}{2 c} \frac{d^{2} \phi_{i}}{d t^{2}}=\widehat{k} 2 \frac{e R^{2} \alpha}{2 c}
$$

since the perturbation contributions in Eq. (37) are in opposite directions.

\section{B. Time-Rate-of-Change of the Mechanical Momentum of the External Charge $q$}

The acceleration of the magnet charges due to the external forces $\overrightarrow{\mathfrak{F}}\left(\phi_{i}\right)=\widehat{\phi}_{i} \mathfrak{F}$ leads to a force $\mathbf{F}_{\text {on } q}=q \mathbf{E}_{\mu}$ on the external charge $q$, where $\mathbf{E}_{\mu}$ involves the acceleration terms of the electric field given in Eq. (3). Since there is already a factor of $q$ in the force $\mathbf{F}_{\text {on } q}=q \mathbf{E}_{\mu}$, we can calculate this force through first order in $q$ while using the the unperturbed positions 
of the magnet particles and the unperturbed acceleration $\alpha$ in Eq. (37),

$$
\begin{aligned}
\mathbf{F}_{\text {on } q} & =\sum_{i=1}^{N} \frac{-q e}{2 c^{2}} R \alpha\left(\frac{\widehat{\phi}_{0 i}}{\left|\mathbf{r}_{0 i}-\mathbf{r}_{q}\right|}+\frac{\widehat{\phi}_{0 i} \cdot\left(\mathbf{r}_{0 i}-\mathbf{r}_{q}\right)\left(\mathbf{r}_{0 i}-\mathbf{r}_{q}\right)}{\left|\mathbf{r}_{0 i}-\mathbf{r}_{q}\right|^{3}}\right) \\
& =\sum_{i=1}^{N} \frac{-q e}{2 c^{2}} R \alpha\left[\frac{\widehat{\phi}_{0 i}}{r_{q}}\left(1+\frac{\mathbf{r}_{0 i} \cdot \mathbf{r}_{q}}{r_{q}^{2}}\right)+\frac{\left(-\widehat{\phi}_{0 i} \cdot \mathbf{r}_{q}\right)\left(\mathbf{r}_{0 i}-\mathbf{r}_{q}\right)}{r_{q}^{3}}\left(1+3 \frac{\mathbf{r}_{0 i} \cdot \mathbf{r}_{q}}{r_{q}^{2}}\right)\right] \\
& =\sum_{i=1}^{N} \frac{-q e}{2 c^{2}} R \alpha\left[\frac{\widehat{\phi}_{0 i}\left(\mathbf{r}_{0 i} \cdot \mathbf{r}_{q}\right)-\left(\widehat{\phi}_{0 i} \cdot \mathbf{r}_{q}\right) \mathbf{r}_{0 i}}{r_{q}^{3}}\right]=\sum_{i=1}^{N} \frac{-e}{2 c^{2}} R \frac{d^{2} \phi}{d t^{2}}\left[\mathbf{r}_{0 i} \times \widehat{\phi}_{0 i}\right] \times \frac{\widehat{r}_{q}}{r_{q}^{2}} \\
& =\sum_{i=1}^{N} \frac{-e}{2 c^{2}} R^{2} \alpha \frac{\widehat{\phi}_{q}}{r_{q}^{2}}=-\widehat{\phi}_{q} \frac{q e R^{2} \alpha}{c^{2} r_{q}^{2}}
\end{aligned}
$$

where we have used $\mathbf{r}_{01}=-\mathbf{r}_{02}, \widehat{\phi}_{01}=-\widehat{\phi}_{02}$, and $\left(\widehat{r}_{0 i} \times \widehat{\phi}_{0 i}\right) \times \widehat{r}_{q}=\widehat{k} \times \widehat{r}_{q}=\widehat{\phi}_{q}$, and there is no need for averaging over the initial angel $\theta_{0}$. This is the same result which we would obtain by calculating the electric field from the changing magnetic moment of the two-moving-particle magnet, given in Eq. (42). Thus the changing magnetic moment gives a force $\mathbf{F}_{\text {on } q}$ on the external charge producing a changing mechanical momentum given by

$$
\frac{d \mathbf{P}_{q}^{\text {mechanical }}}{d t}=\mathbf{F}_{\text {onq }}=-\frac{q}{c} \frac{d \vec{\mu}}{d t} \times \frac{\widehat{r}_{q}}{r_{q}^{2}}=-\frac{q}{c} \widehat{k} 2 \frac{e R^{2} \alpha}{2 c} \times \frac{\widehat{r}_{q}}{r_{q}^{2}}=-\widehat{\phi}_{q} \frac{q e R^{2} \alpha}{c^{2} r_{q}^{2}}
$$

\section{Time-Rate-of-Change of External Electromagnetic Momentum}

The applied force $\overrightarrow{\mathfrak{F}}\left(\phi_{i}\right)=\widehat{\phi}_{i} \mathfrak{F}\left(\phi_{i}\right)$ also changes both the internal and external electromagnetic momentum. The external electromagnetic momentum $\mathbf{P}_{q-\mu}^{e m}$ is given in Eq. (11); it's rate of change for the two-particle model is

$$
\frac{d \mathbf{P}_{q-\mu}^{e m}}{d t}=\frac{d \vec{\mu}}{d t} \times \frac{q \widehat{r}_{q}}{c r_{q}^{2}}=\frac{q}{c} \widehat{k} 2 \frac{e R^{2} \alpha}{2 c} \times \frac{\widehat{r}_{q}}{r_{q}^{2}}=\widehat{\phi}_{q} \frac{q e R^{2} \alpha}{c^{2} r_{q}^{2}}
$$

\section{Conserved Canonical Momentum for the External Charge $q$}

The rate of change of the external electromagnetic momentum $d \mathbf{P}_{q-\mu}^{e m} / d t$ given in Eq. (45) is just the negative of the rate of change of the mechanical momentum $d \mathbf{P}_{q}^{\text {mechanical }} / d t=\mathbf{F}_{\text {on } q}$ appearing in Eq. (44). Thus on the application of the external forces $\overrightarrow{\mathfrak{F}}\left(\phi_{i}\right)=\widehat{\phi_{i}} \mathfrak{F}\left(\phi_{i}\right)$ to the magnet, we have the conservation of the canonical momentum $\mathbf{P}_{q}^{\text {canonical }}=\mathbf{P}_{q}^{\text {mechanical }}+\mathbf{P}_{q-\mu}^{e m}$ associated with the charge $q$. Conservation of this canonical momentum was indicated in the article by Coleman and Van Vleck. [2] Both of these contribution to the canonical momentum 
depend upon the unperturbed positions of the charges in the magnet and so are familiar in textbook electromagnetism (which does not consider the possibility of perturbations for the currents of the magnet).

\section{E. Time-Rate-of-Increase of the Mechanical Momentum of the Moving Magnet}

\section{Charges}

In order to calculate the time-rate-of-increase of the mechanical momentum of the moving magnet charges, we introduce the angular acceleration in Eq. (37) into the expression for the time rate of change of the linear momentum of each particle but now include the slight perturbation $\eta_{i}$ of the location of each particle due to the polarization caused by the external

charge $q$. Thus using Eqs. (24) and (30), the average mechanical momentum $\left\langle\mathbf{P}_{\mu}^{\text {mechanical }}\right\rangle$ of the magnet charges changes as

$$
\begin{aligned}
\left\langle\frac{d \mathbf{P}_{\mu}^{\text {mechanical }}}{d t}\right\rangle & =\left\langle\sum_{i=1}^{2} \widehat{\phi}_{i} m R \frac{d^{2} \phi_{i}}{d t^{2}}\right\rangle=\left\langle\sum_{i=1}^{2} \widehat{\phi}_{i} m R \alpha\left[1+\frac{1}{\omega_{0}} \frac{d \eta_{i}}{d t}\right]\right\rangle \\
& =m R \alpha \sum_{i=1}^{2}\left\langle\phi_{0 i}-\widehat{r}_{0 i} \eta_{i}+\frac{\widehat{\phi}_{0 i}}{\omega_{0}} \frac{d \eta_{i}}{d t}\right\rangle=0
\end{aligned}
$$

where we have noted that

$$
\left\langle\widehat{\phi}_{0 i}\right\rangle=\left\langle\widehat{r}_{0 i}\right\rangle=0
$$

and

$$
\left\langle\widehat{r}_{0 i} \eta_{i}\right\rangle=\frac{\widehat{\phi}_{q} 2 q R^{2}}{e r_{q}^{2}}=\left\langle\frac{\widehat{\phi}_{0 i}}{\omega_{0}} \frac{d \eta_{i}}{d t}\right\rangle
$$

\section{F. Time-Rate-of-Change of Internal Electromagnetic Momentum}

The internal electromagnetic linear momentum is that given in Eq. (32). For a twoparticle magnet, the time-rate-of-change of this internal electromagnetic momentum is

$$
\frac{d \mathbf{P}_{\mu}^{e m}}{d t}=\sum_{i=1}^{2} \frac{e^{2}}{2 c^{2}\left|\mathbf{r}_{i}-\mathbf{r}_{j \neq i}\right|}\left(\mathbf{a}_{j \neq i}+\frac{\mathbf{a}_{j \neq i} \cdot\left(\mathbf{r}_{i}-\mathbf{r}_{j \neq i}\right)\left(\mathbf{r}_{i}-\mathbf{r}_{j \neq i}\right)}{\left|\mathbf{r}_{i}-\mathbf{r}_{j \neq i}\right|^{2}}\right)
$$

since the velocity of the charges is very small and we are ignoring terms in $v_{i}^{2}$. Only the tangential acceleration $\widehat{\phi}_{0 i}\left(\widehat{\phi}_{0 i} \cdot \mathbf{a}_{i}\right)=\widehat{\phi}_{0 i} R\left(d^{2} \phi_{i} / d t^{2}\right)$ needs to be considered, so that Eq. (49) 
becomes through first order in the perturbation due to $q$,

$$
\begin{aligned}
\left\langle\frac{d \mathbf{P}_{\mu}^{e m}}{d t}\right\rangle & =\left\langle\sum_{i=1}^{2} \frac{e^{2}}{2 c^{2}\left|\mathbf{r}_{i}-\mathbf{r}_{j \neq i}\right|}\left(\widehat{\phi}_{j \neq i}+\frac{\widehat{\phi}_{j \neq i} \cdot\left(\mathbf{r}_{i}-\mathbf{r}_{j \neq i}\right)\left(\mathbf{r}_{i}-\mathbf{r}_{j \neq i}\right)}{\left|\mathbf{r}_{i}-\mathbf{r}_{j \neq i}\right|^{2}}\right) R \frac{d^{2} \phi_{j \neq i}}{d t^{2}}\right\rangle \\
& \approx \frac{e^{2}}{2 c^{2}(2)} \sum_{i=1}^{2}\left\langle\left(\widehat{\phi}_{j \neq i}+\frac{1}{4}\left(\widehat{\phi}_{j \neq i} \cdot \widehat{r}_{i}\right)\left(\widehat{r}_{i}-\widehat{r}_{j \neq i}\right)\right) \alpha\left[1+\frac{1}{\omega_{0}} \frac{d \eta_{j}}{d t}\right]\right\rangle \\
& =\frac{e^{2} \alpha}{4 c^{2}} \sum_{i=1}^{2}\left\langle\left(\widehat{\phi}_{0 j \neq i}-\widehat{r}_{0 j \neq i} \eta_{j \neq i}\right)+\left[-\eta_{i}\right]\left[\widehat{r}_{0 i}\right]+\frac{\widehat{\phi}_{0 j \neq i}}{\omega_{0}} \frac{d \eta_{j}}{d t}\right\rangle \\
& =\frac{e^{2} \alpha}{4 c^{2}} \sum_{i=1}^{2}\left\langle-2 \widehat{r}_{0 i} \eta_{i}+\frac{\widehat{\phi}_{0 i}}{\omega_{0}} \frac{d \eta_{i}}{d t}\right\rangle=-\frac{e q R^{2} \alpha}{c^{2} r_{q}^{2}} \widehat{\phi}_{q}
\end{aligned}
$$

where we have used Eqs. (27), (47), and (48). But this result for the time rate of change of the average internal electromagnetic momentum $\left\langle d \mathbf{P}_{\mu}^{e m} / d t\right\rangle$ in Eq. (50) is indeed equal in magnitude and opposite in sign from the time rate of change of the external electromagnetic momentum $d \mathbf{P}_{q-\boldsymbol{\mu}}^{e m} / d t$ in Eq. (45), so that the time rate of change of the total electromagnetic momentum vanishes on average

$$
\frac{d\left\langle\mathbf{P}_{\text {total }}^{e m}\right\rangle}{d t}=\frac{d \mathbf{P}_{q-\mu}^{e m}}{d t}+\left\langle\frac{d \mathbf{P}_{\mu}^{e m}}{d t}\right\rangle=0
$$

\section{G. External Tangential Forces}

Next we wish to consider the sum over the external forces which act on the system of the magnet and external point charge. From Eq. (41), the sum of the forces causing the change in the magnetic moment is given by

$$
\begin{aligned}
\left\langle\mathbf{F}_{\text {on } \mu}^{\text {tangential }}\right\rangle & =\sum_{i=1}^{2}\left\langle\overrightarrow{\mathfrak{F}}\left(\phi_{i}\right)\right\rangle=\sum_{i=1}^{2}\left\langle\widehat{\phi}_{i} \mathfrak{F}_{i}\right\rangle \\
& =\sum_{i=1}^{2}\left\langle\widehat{\phi}_{i} m R \alpha\left\{\left[1+\frac{1}{\omega_{0}} \frac{d \eta_{i}}{d t}\right]-\frac{e^{2}}{4 c^{2}} \alpha\left[1+\frac{1}{\omega_{0}} \frac{d \eta_{j \neq i}}{d t}\right]\right\}\right\rangle \\
& =\sum_{i=1}^{2} m R \alpha\left\{\left\langle\widehat{\phi}_{i}+\frac{\widehat{\phi}_{i}}{\omega_{0}} \frac{d \eta_{i}}{d t}\right\rangle-\frac{e^{2}}{4 c^{2}} \alpha\left\langle\widehat{\phi}_{i}-\frac{\widehat{\phi}_{i}}{\omega_{0}} \frac{d \eta_{i}}{d t}\right\rangle\right\}
\end{aligned}
$$

Now the first term in brackets vanishes

$$
\left\langle\widehat{\phi}_{i}+\frac{\widehat{\phi}_{i}}{\omega_{0}} \frac{d \eta_{i}}{d t}\right\rangle=0
$$


since this involves the same averages as appeared in Eq. (46). However, the second term involves contributions which add to give

$$
\left\langle\widehat{\phi}_{i}-\frac{\widehat{\phi}_{i}}{\omega_{0}} \frac{d \eta_{i}}{d t}\right\rangle=\left\langle\phi_{0 i}-\widehat{r}_{0 i} \eta_{i}-\frac{\widehat{\phi}_{0 i}}{\omega_{0}} \frac{d \eta_{i}}{d t}\right\rangle=-2 \frac{\widehat{\phi}_{q} 2 q R^{2}}{e r_{q}^{2}}
$$

Therefore the sum of the tangential forces $\left\langle\mathbf{F}_{\text {on } \mu}^{\text {tangential }}\right\rangle$ causing the change in the magnetic moment is

$$
\left\langle\mathbf{F}_{\text {on } \mu}^{\text {tangential }}\right\rangle=\sum_{i=1}^{2}\left\langle\overrightarrow{\mathfrak{F}}\left(\phi_{i}\right)\right\rangle=-\frac{e^{2}}{4 c^{2}} \alpha 2\left(-2 \frac{\widehat{\phi}_{q} 2 q R^{2}}{e r_{q}^{2}}\right)=2 \frac{e q R^{2} \alpha}{c^{2} r_{q}^{2}} \widehat{\phi}_{q}
$$

\section{H. Radial Forces of Constraint}

There is one additional and crucial set of external forces, those which act on the system to maintain the orbital motion of the moving magnet charges. In Eq. (40), we saw the tangential contribution of the force of each magnet charge on the other due to the acceleration arising from the forces $\overrightarrow{\mathfrak{F}}\left(\phi_{i}\right)$. The acceleration of the magnet charges $e_{i}$ also causes a radial component of force of each charge on the other. This radial component of force must be balanced by external radial forces of constraint which keep the magnet charges moving in the circular orbit. Thus we have a net force introduced by the radial forces of constraint given by

$$
\mathbf{F}_{\text {on } \mu}^{\text {radial }}=-\sum_{i=1}^{2} \widehat{r}_{i} \widehat{r}_{i} \cdot\left[e \mathbf{E}_{j \neq i}\left(\mathbf{r}_{i}\right)+e \mathbf{E}_{q}\left(\mathbf{r}_{i}\right)\right]
$$

where the centripetal accelerations $\omega_{i}^{2} R$ of the charges are negligible for small $\omega_{0}$. Now the sum of the electrostatic forces on the charges of the neutral magnet will vanish except for the electrostatic dipole force of the distant external charge $q$ on the polarized magnet, which we neglect as higher order in both $q$ and also $R / r_{q}$. However, acceleration fields and forces

arise when the forces $\overrightarrow{\mathfrak{F}}\left(\phi_{i}\right)$ act. Thus we must have the additional net force of constraint 
which balances the electric acceleration forces,

$$
\begin{aligned}
\left\langle\mathbf{F}_{\text {on } \mu}^{\text {radial }}\right\rangle & =-\left\langle\sum_{i=1}^{2} \widehat{r}_{i} \widehat{r}_{i} \cdot\left[\frac{-e^{2}}{2 c^{2}} R \frac{d^{2} \phi_{j}}{d t^{2}}\left(\frac{\widehat{\phi}_{j \neq i}}{\left|\mathbf{r}_{i}-\mathbf{r}_{j \neq i}\right|}+\frac{\widehat{\phi}_{j \neq i} \cdot\left(\mathbf{r}_{i}-\mathbf{r}_{j \neq i}\right)\left(\mathbf{r}_{i}-\mathbf{r}_{j \neq i}\right)}{\left|\mathbf{r}_{i}-\mathbf{r}_{j \neq i}\right|^{3}}\right)\right]\right\rangle \\
& =\left\langle\sum_{i=1}^{2} \widehat{r}_{i}\left[\frac{e^{2}}{2 c^{2}} R \frac{d^{2} \phi_{j}}{d t^{2}}\left(\frac{\widehat{r}_{i} \cdot \widehat{\phi}_{j \neq i}}{\left|\mathbf{r}_{i}-\mathbf{r}_{j \neq i}\right|}+\frac{\left(\widehat{\phi}_{j \neq i} \cdot \widehat{r}_{i}\right) \widehat{r}_{i} \cdot\left(\widehat{r}_{i}-\widehat{r}_{j \neq i}\right) R^{2}}{\left|\mathbf{r}_{i}-\mathbf{r}_{j \neq i}\right|^{3}}\right)\right]\right\rangle \\
& =\left\langle\sum_{i=1}^{2} \widehat{r}_{i}\left[\frac{e^{2}}{2 c^{2}} R \frac{d^{2} \phi_{j}}{d t^{2}}\left(\frac{\eta_{j \neq i}-\eta_{i}}{2 R}+\frac{\left(\eta_{j \neq i}-\eta_{i}\right) \widehat{r}_{i} \cdot\left(\widehat{r}_{i}-\widehat{r}_{j \neq i}\right) R^{2}}{(2 R)^{3}}\right)\right]\right\rangle \\
& =\left\langle\sum_{i=1}^{2} \widehat{r}_{0 i}\left[\frac{e^{2}}{2 c^{2}} R \alpha\left(\frac{-2 \eta_{i}}{2 R}+\frac{\left(-2 \eta_{i}\right)(2) R^{2}}{(2 R)^{3}}\right)\right]\right\rangle \\
& =\frac{e^{2} \alpha}{2 c^{2}} \sum_{i=1}^{2}\left\langle-\widehat{r}_{0 i} \eta_{i}+\frac{\left(-\widehat{r}_{0 i} \eta_{i}\right)}{(2)}\right\rangle=\frac{-3 e^{2} \alpha}{4 c^{2}} \sum_{i=1}^{2}\left\langle\widehat{r}_{0 i} \eta_{i}\right\rangle \\
& =\frac{-3 e^{2} \alpha}{4 c^{2}} 2\left[\frac{2 q R^{2}}{e r_{q}^{2}} \widehat{\phi}_{q}\right]=-3 \frac{q R^{2} e \alpha}{r_{q}^{2} c^{2}} \widehat{\phi}_{q}
\end{aligned}
$$

where we have used Eq. (27) and have retained terms only through first order in the angular perturbation $\eta_{i}$.

\section{Conservation of Linear Momentum}

The average sum of the external forces on the system of the magnet and the external charge $q$ involves adding both $\left\langle\mathbf{F}_{\text {on } \mu}^{\text {tangential }}\right\rangle$ given in Eq. (154) and $\left\langle\mathbf{F}_{\text {on } \mu}^{\text {radial }}\right\rangle$ given in Eq. (56). Thus we have

$$
\left\langle\mathbf{F}_{\text {on } \mu}^{\text {tangential }}+\mathbf{F}_{\text {on } \mu}^{\text {radial }}\right\rangle=2 \frac{e q R^{2} \alpha}{c^{2} r_{q}^{2}} \widehat{\phi}_{q}-3 \frac{q R^{2} e \alpha}{r_{q}^{2} c^{2}} \widehat{\phi}_{q}=-\frac{q R^{2} e \alpha}{r_{q}^{2} c^{2}} \widehat{\phi}_{q}
$$

The average sum of the rates of change of momentum delivered to the magnet-point charge system in Eqs. (44), (45), (46), and (50) is given by

$$
\begin{gathered}
\left\langle\frac{d \mathbf{P}_{q}^{\text {mechanical }}}{d t}+\frac{d \mathbf{P}_{q-\mu}^{e m}}{d t}+\frac{d \mathbf{P}_{\mu}^{\text {mechanical }}}{d t}+\frac{d \mathbf{P}_{\mu}^{e m}}{d t}\right\rangle \\
=-\frac{q R^{2} e \alpha}{r_{q}^{2} c^{2}} \widehat{\phi}_{q}+\frac{q R^{2} e \alpha}{r_{q}^{2} c^{2}} \widehat{\phi}_{q}+0-\frac{e q R^{2} \alpha}{c^{2} r_{q}^{2}} \widehat{\phi}_{q}=-\frac{q R^{2} e \alpha}{r_{q}^{2} c^{2}} \widehat{\phi}_{q}
\end{gathered}
$$

But then indeed we find conservation of linear momentum for the system of the magnet and the point charge since the average external force in Eq. (57) matches the rate of change

of total momentum in Eq. (58). Since the canonical momentum $\mathbf{P}_{q}^{\text {canonical }}=\mathbf{P}_{q}^{\text {mechanical }}+$ 
$\mathbf{P}_{q-\mu}^{e m}$ of the charge $q$ does not change in time, the average external forces (which act only on the magnet) give the time rate of change of the canonical momentum of the magnet. Another way of viewing the situation is to note that the total electromagnetic momentum of the system vanishes and that the external forces applied to the magnet account for the change in the mechanical momentum of the external charge $q$. There is no problem with the conservation of momentum.

\section{SUMMARY DISCUSSION}

Although Coleman and Van Vleck[2] provide many valuable insights into the behavior of the system consisting of a magnet and a point charge, they point to relativistic hidden mechanical momentum as the crucial element providing the momentum balance required by special relativity. In a footnote, they mention the example of a single charged particle moving in a closed orbit in the electrostatic field of the external charge $q$. This singleparticle model for a magnet appears in a monograph on the electrodynamics of moving media. 16] Also a standard undergraduate textbook presents the equivalent of this singleparticle model by assuming that there is no interaction among the charges in the magnet. [24] However, the single-particle model (or equivalently a non-interacting-charge model) for the magnet gives an entirely false description of a multiparticle magnetic system. Just as a single-particle description (or non-interacting particle description) of the self-inductance of a circuit involves the mass of the current carriers and so gives a false impression regarding the behavior of the electromagnetic circuit, so the single-particle description of a magnet gives a false description of the interaction of an interacting-multiparticle magnet and an external point charge.

There seem to be three crucial aspects which go unappreciated in the literature. First of all, contrary to the claim of Coleman and Van Vleck, the polarization of the magnet by the external charge is not something which may be neglected. Now it is true that the electrostatic forces between the magnet and the distant point charge which arise from the polarization can be neglected as small. However, the electrostatic polarization is crucial in influencing the behavior of the magnet. Second, the magnet itself can contain internal electromagnetic momentum under the influence of the electrostatic polarization caused by the

external charge. The external electromagnetic momentum involving the electric field of the 
external charge and the magnetic field of the magnet is a familiar aspect of electromagnetic theory which appears in the textbooks. However, the idea of an internal electromagnetic momentum where both the electric and magnetic fields arise from charges within the magnet does not seem to be recognized. Coleman and Van Vleck correctly refer to the requirement of special relativity that the total momentum of a steady-state system must vanish, and therefore they conclude that the magnet must contain some internal momentum; however, they mention only the possibility of relativistic mechanical internal momentum. For a magnet consisting of a single moving charge (or many non-interacting charges), the internal momentum is indeed mechanical. For a magnet consisting of a few interacting particles, the internal momentum will involve both mechanical and electromagnetic momentum. For an interacting-multiparticle magnet or for a few-interacting-particle magnet of very lowvelocity charges, the internal electromagnetic momentum dominates the internal mechanical momentum of the magnet, and this leads to qualitative changes in the momentum balance of the system consisting of a magnet and a point charge. Third, for a steady-state situation when the induced charge distribution of the magnet is essentially electrostatic, the internal electromagnetic momentum is equal in magnitude and opposite in direction from the familiar external electromagnetic momentum. Thus the total electromagnetic momentum of the system is zero. 25$]$

Acknowledgement

Although in this article, I have criticized a blunder made by S. Coleman and J. H. Van Vleck, I am vastly in their debt. Their analysis of the Shockley-James paradox was invaluable to my understanding of the interaction of a magnet and a charged particle.

[1] W. Shockley and R. P. James, “Try simplest cases' discovery of 'hidden momentum' forces on 'magnetic currents,"' Phys. Rev. Lett. 18, 876-879 (1967).

[2] S. Coleman and J. H. Van Vleck, "Origin of 'hidden momentum forces' on magnets," Phys. Rev. 171, 1370-1375 (1968).

[3] D. J. Griffiths, "Resource Letter EM-1: Electromagnetic Momentum," Am. J. Phys. 80, 7-18 (2012).

[4] J. D. Jackson, Classical Electrodynamics 3rd edn (Wiley, New York 1999), pp.189, 618. 
[5] D. J. Griffiths, Introduction to Electrodynamics 3rd edn (Prentice-Hall, Upper Saddle River,NJ 1999), pp. $357,361,520-521$.

[6] L. Vaidman, "Torque and force on a magnetic dipole," Am. J. Phys. 58, 978-983 (1990).

[7] Y. Aharonov, P. Pearle, and L. Vaidman, "Comment on 'proposed Aharonov-Casher effect: another example of an Aharonov-Bohm effect arising from a classical lag,"' Phys. Rev. 115, 485-491 (1988).

[8] Y. Aharonov and D. Rohrlich, Quantum Paradoxes: Quantum Theory for the Perplexed, (Wiley-VCH, Weinheim 2005).

[9] See the list in reference 27 of T. H. Boyer, "Examples and comments related to relativity controversies," Am. J. Phys. 80, 962-971 (2012).

[10] M. Mansuripur, "Trouble with the Lorentz law of force: Incompatibility with special relativity and momentum conservation," Phys. Rev. Lett. 108, 193901 (2012).

[11] T. H. Boyer, "Self-Inductance and the Mass of Current Carriers in a Circuit," submitted for publication.

[12] See ref. 4, Section 12.6, pp. 596-598.

[13] L. Page and N. I. Adams, "Action and reaction between moving charges," Am. J. Phys. 13, 141-147 (1945).

[14] T. H. Boyer, "Illustrations of the relativistic conservation law for the center of energy," Am. J. Phys. 73, 953-961 (2005), Eq. (14). The generator of Lorentz transformations is the system energy times the center of energy.

[15] See for example, ref. 4, p. 286, problem 6.5.

[16] P. Penfield and H. A. Haus, Electrodynamics of Moving Media (MIT Press, Cambridge, MA 1967) p. 215.

[17] See footnote 9 in ref. 2.

[18] T. H. Boyer, "Interaction of a Magnet and a Point Charge: Unrecognized Internal Electromagnetic Momentum Eliminates the Myth of Hidden Mechanical Momentum," submitted for publication.

[19] See for example, T. H. Boyer, "Classical Electromagnetic Interaction of a Charged Particle with a Constant-Current Solenoid," Phys. Rev. 8, 1667-1679 (1973).

[20] W. H. Furry, "Examples of Momentum Distributions in the Electromagnetic Field and in Matter," Am. J. Phys. 37, 621-636 (1969). 
[21] See for example, F. S. Johnson, B. L. Cragin, and R. R. Hodges, "Electromagnetic momentum density and the Poynting vector in static fields," Am. J. Phys.62, 33-41 (1994).

[22] See for example, T. H. Boyer, "Interaction of a Point Charge and a Magnet: Comments on 'Hidden Mechanical Momentum Due to Hidden Nonelectromagnetic Forces,' " arXiv:0708.3367 (2007).

[23] In this two-particle model, the acceleration fields tend to assist the external forces in accelerating the particles, rather than holding the particles back. Thus in Eq. (43), we see the appearance of $m R-e^{2} /\left(2 c^{2}\right)$ with a minus rather than a plus sign. The induced emf acting on the particles of the magnet in this case is actually in the opposite direction from that expected for a multiparticle magnetic moment. As pointed out in ref. 11, a minimum of four charged particles is required before the induced emf opposes the acceleration and gives a Faraday induction field within the magnet in the direction expected for a changing multiparticle magnetic moment. The conservation laws are valid for the two-particle magnet, but there are some surprising aspects.

[24] D. J. Griffiths, Introduction to Electrodynamics 4th edn (Pearson, Boston, MA 2013), pp. 547-549.

[25] The present article is the third in a series of four articles by T. H. Boyer using the magnet model consisting of mutually-interacting point charges moving on a circular path. Article 1: "Self-Inductance and the Mass of Current Carriers in a Circuit." Article 2: "Interaction of a Magnet and a Point Charge: Unrecognized Internal Electromagnetic Momentum Eliminates the Myth of Hidden Mechanical Momentum." Article 4: "Classical interaction of a Magnet and a Point Charge: The Classical Electromagnetic Forces Responsible for the AharonovBohm Phase Shift." 